\title{
Behavioral Findings during Recovery after Experimental Stroke in Monkeys -Assessment with Modified Hand Performance Test-
}

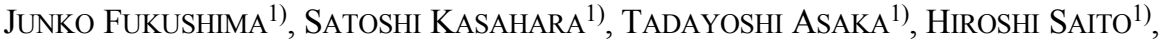 \\ MASANORI YAMANAKA ${ }^{1)}$ \\ ${ }^{1)}$ Division of Physical Therapy, Department of Health Sciences, Hokkaido University \\ School of Medicine: West 5 North 12, Kitaku, Sapporo 060-0812, Japan. \\ TEL \& FAX +81 11-706-3329, E-mail: jf002@cme.hokudai.ac.jp
}

\begin{abstract}
It is still unclear how rehabilitative intervention assists recovery from strokes. In order to address this question, we made an animal model to examine the recovery process after experimental strokes. The forelimb movements in 2 monkeys retrieving food from a well were analyzed with a videotape recording. A small infarction by coagulation of blood vessels was made in the forelimb areas after mapping of the primary motor areas. The recovery process was evaluated by measuring the time required for taking food. We used the modified Klüver board with the small diameter in order to restrict the monkeys' compensatory movements such as pronation or supination. After the infarction, the monkeys could not use their impaired hands for the first 2 days. Failure to retrieve food and compensatory movements such as involvement of other joints were often observed during the first week. However, the forelimb function of both monkeys showed no significant impairments on the 6th week, compared to the results before the infarction. Throughout the experiments, the monkeys showed no supination or pronation of the forearm. These results suggest that this animal model with the modified Klüver board may be useful for the evaluation of motor deficit.
\end{abstract}

Key words: Animal model, Cerebral infarction, Recovery process

(This article was submitted Aug. 16, 2006, and was accepted Sep. 19, 2006)

\section{INTRODUCTION}

Although various rehabilitative therapeutic exercises are reported to improve the function of patients after strokes from various aspects ${ }^{1-3)}$, how they assist recovery from dysfunction has not been elucidated. It has been reported that rehabilitative therapeutic exercises have a certain effect on the reorganization of the nervous system of the cerebral cortex and cerebellum ${ }^{4,5)}$. Nudo et al. ${ }^{6,7)}$ showed the effects of exercises on somatotopic reorganization by means of microstimulation in the primary motor cortex of the monkeys that had received experimental procedures of cerebral infarction. Xerri et al. ${ }^{8)}$ reported the changes of somatotopy in the areas of $3 a$ and $3 b$ after the experimental infarction in squirrel monkeys. Both studies showed changes in the areas surrounding the infarction that compensated for the function of the areas with infarction. These studies indicate that rehabilitative therapeutic exercises are involved in the recovery of motor function in stroke patients, suggesting that the regions surrounding the infarction may compensate for the impaired 
function of the infarcted areas.

Barbay et al. indicated that delayed training resulted in an increase of co-contractive movements evoked with microstimulation ${ }^{9)}$. This suggests motor skill training in the chronic stages following stroke may modulate peri-infarct cortical plasticity in a different way. They also observed increase of multi-joint movements in both wrist, forearm and other proximal areas of the upper extremities evoked with microstimulation. This indicates that the reorganization of the brain is different from that induced by the training started earlier. Moreover, these multi-joint responses in the forearms ${ }^{9)}$ showed a negative correlation with the behavioral improvement in clinical evaluation ${ }^{10)}$.

Friel et al. examined precise abnormal multi-joint movements during behavioral recovery after experimental cortical injury in non-human primates ${ }^{11)}$. They reported various kinds of compensatory movement patterns such as pronation and supination of the forearm, in addition to flexion and extension of the wrist and digits to take food pellets. Although precise observation of behavioral recovery may be important for future studies, it may be difficult to evaluate the results quantitatively, if the animals show various kinds of compensatory movement patterns. Moreover, compensatory movements as abnormal patterns in acute hemiparesis should be suppressed for recovery, according to Bobath's methods ${ }^{12)}$.

The purpose of the present study is to evaluate the effects of therapeutic exercises on the recovery of forelimbs after the experimental lesion in the primary motor cortex, and to investigate whether the compensatory movements such as pronation or supination can be suppressed using the modified Klüver board with small diameters and different depths. We also attempted to compare the recovery process of two monkeys that received experimental lesions in either the digit or shoulder area.

\section{MATERIALS AND METHODS}

\section{General procedures}

Two adult male monkeys (Monkey A: Rhesus Monkey $6.4 \mathrm{~kg}$, Monkey B: Japanese Monkey 7.1 $\mathrm{kg}$ ) were used in this study. All experiments were performed in strict compliance with the Guide for the Care and Use of Laboratory Animals (NRC 1997). The procedures described below were evaluated and approved by the Animal Care and
Use Committee of Hokkaido University School of Medicine. All procedures except for surgery were performed in the cages where the monkeys were reared. After examining the hand preference, control data before experimental strokes were taken. Then, under anesthesia, electrophysiological lesions were made in the areas of forelimbs in the primary motor cortex after mapping. After surgery, the monkeys started therapeutic exercises and continued them every day. Except for during the Hand Performance Test, both Monkeys were allowed free access to water and food, without any restriction.

\section{Hand preference testing}

To determine the hand preference, we placed a food pellet on a flat table in front of each monkey's cage. Monkeys could easily reach the food through the window of the cage. We determined hand preference according to which hand was used more often. After determining the dominant hand, a board with wells was placed at the left side of the cage in front of the monkeys, so that the monkeys could use only their dominant hands.

\section{Pre-infarct training procedure}

To obtain the data before and after surgery, and the training of digits, we performed the Hand Performance Test using a modified Klüver board ${ }^{13)}$ (Fig. 1). The modified Klüver board had 5 wells with the same diameters $(30 \mathrm{~mm})$ but with different depths $(10,20,30,40$ and $50 \mathrm{~mm})$, based on the width and length of the monkeys' hands. The reason why we used the wells with the same small diameters is that monkeys are unable to pronate or supinate their forearms in the wells. The food for the test in the present study was a piece of apple cut to 1 cubic $\mathrm{cm}$. To retrieve food, the monkeys had to stretch their dominant forelimbs from a $10 \times 10 \mathrm{~cm}$ window located at the left side of the cage in front of them. At first, food was placed on a flat table. Then they were trained to take it from the shallowest well of $10 \mathrm{~mm}$ depth. After they learned to take food from it consistently, they took it from the well with the depth of $20,30,40$ and $50 \mathrm{~mm}$, one after another.

The baseline of the time required for retrieval was determined by the averaged time for taking it from each well for 3 days before the surgery. In 8 of the 63 sessions, recordings were made with a videotape system (Digital Video Cassette Recorder, DCR- 

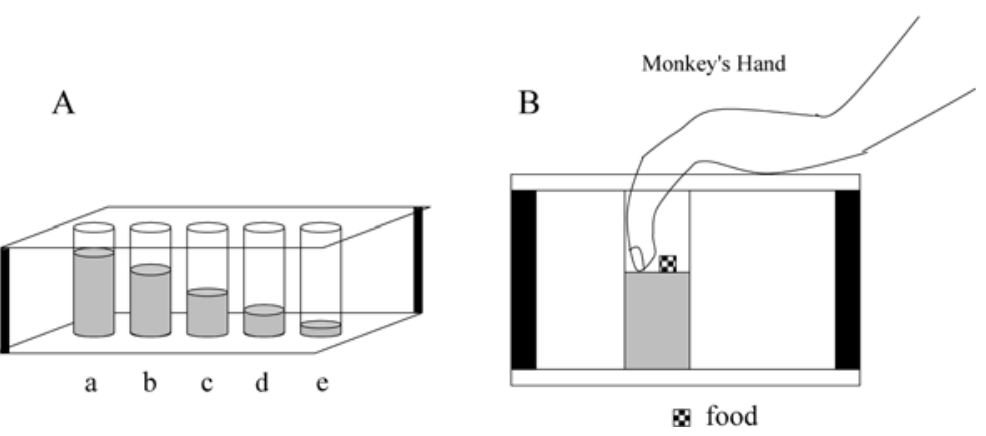

Fig. 1. Hand performance test. A. Plexiglas board (modified Klüver board) containing five wells was mounted on the left side in front of the animal's cage. The depth of each well was $10 \mathrm{~mm}$ (a), $20 \mathrm{~mm}$ (b), 30 $\mathrm{mm}$ (c), $40 \mathrm{~mm}$ (d), and $50 \mathrm{~mm}$ (e). The diameter of each well was $30 \mathrm{~mm}$. B. A single small piece of food was placed in a well, from which the monkeys retrieved it.

TRV900 Sony) with a sampling rate of 30 frames/ sec to examine the joint movements of the forelimbs. In the other sessions, an experimenter measured the time required for retrieving food, using a stopwatch. Although the usual training sessions consisted of 30 minutes, the training was discontinued when the monkeys did not take food for more than 5 minutes.

\section{Electrophysiological mapping and the lesion for experimental stroke}

Before making experimental lesions, we applied intracortical electrical stimulation to determine the hand area of the primary motor cortex. After we confirmed that both monkeys were able to retrieve food from all of the wells, the monkeys were sedated by ketamine $(6-7 \mathrm{mg} / \mathrm{kg})$. They were anesthetized by halothane/nitrous oxide and an intraperitoneal injection of pentobarbital sodium $(2.0 \mathrm{mg} / \mathrm{kg})$. Under aseptic conditions, from a small opening, a tungsten electrode with an impedance of $0.8 \sim 1.0 \mathrm{M} \Omega$ was inserted into the monkeys' hand areas of the right primary motor cortex (M1; A12 L18). At each site, current was delivered through the electrode whose tip was positioned 1,500-1,900 $\mu \mathrm{m}$ below the cortical surface. The strength of the current was set $50 \mu \mathrm{A}(0.2 \mathrm{~ms}, 300 \mathrm{~Hz}, 11$ cathodal pulse), at first. However, because of the effects of anesthesia, we increased the current to $200 \mu \mathrm{A}$ until we could get responses. We determined the stimulated point as "no response", when we could not get any responses despite applying more than $200 \mu \mathrm{A}$.
Acute ischemic lesions were made by a microcoagulater to pinch the blood vessels at the digit areas in M1 in Monkey B, and the shoulder area in Monkey A.

\section{Assessment of motor performance on the Klüver board}

Motor performances were analyzed with the video recording, to examine the following points: a) how long it took to retrieve food (the beginning was determined as the time when a part of the hand entered the well, and the end was the time for leaving the whole part of the hand from the well); b) how many times each joint (e.g. digit, wrist, elbow and shoulder) was moving to take food; c) failure, defined as when the monkeys could not take food despite putting their hands into the well. The failure percentage was calculated as the number of failures divided by the number of the trials to take food $(\times 100)$.

\section{Statistical analysis}

The required time for each well was compared with one-way and two-way ANOVA. Fisher's PLSD was also used for testing the significance level $(\mathrm{p}<0.05)$.

\section{RESULTS}

\section{Hand performance in normal monkeys}

The movement pattern of successfully taking food was relatively constant. During the hand preference test, Monkey A used only the left hand. 

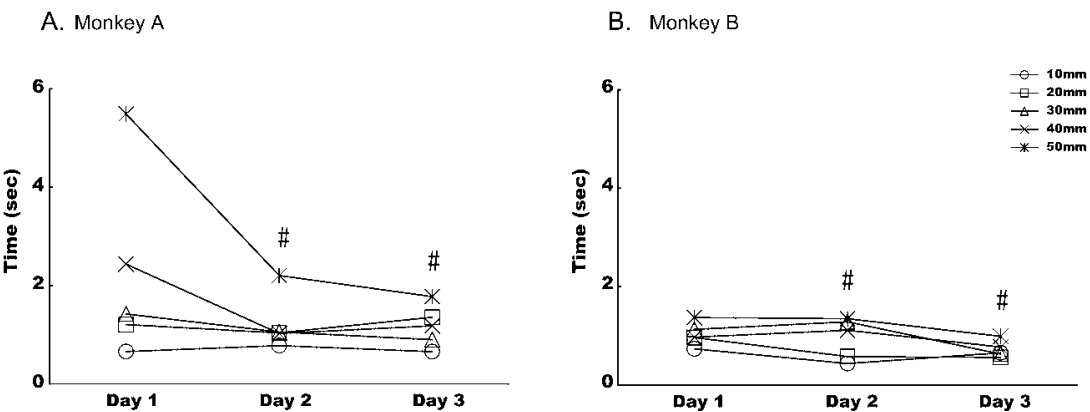

Fig. 2. The time required for retrieving the food in the Hand Performance Test with the dominant hand before experimental lesion. A. Monkey A showed significant differences for both depths and days (\#). B. Monkey B showed significant differences for both depths and days (\#).

Although Monkey B took food by his either left or right hand at first, he used only his left hand, after he repeated practice. From these findings, we determined that both monkeys were left-handed.

Figure 2 shows the average time required for retrieving food in hand performance tests before surgery. The average ( \pm standard deviation) time for the 2 monkeys was $0.67 \pm 0.27 \mathrm{sec}$ for the well of $10 \mathrm{~mm}$ depth, $1.04 \pm 0.95 \mathrm{sec}$ for $20 \mathrm{~mm}, 1.05 \pm$ $0.65 \mathrm{sec}$ for $30 \mathrm{~mm}, 1.25 \pm 0.74 \mathrm{sec}$ for $40 \mathrm{~mm}$, and $2.16 \pm 1.68 \mathrm{sec}$ for $50 \mathrm{~mm}$. Monkey A showed a significant difference for days $\left(\mathrm{F}_{2,101}=13.9\right.$, $\mathrm{p}<0.001)$ and depth $\left(\mathrm{F}_{4,104}=22.6, \mathrm{p}<0.001\right)$.

Monkey $\mathrm{B}$ needed a significantly shorter time to retrieve food than Monkey $A$ at the beginning $\left(\mathrm{F}_{1}\right.$, $\left.{ }_{153}=18.5, \mathrm{p}<0.001\right)$. He also showed a significant difference in the results between the 1st day and the other days $\left(\mathrm{F}_{2,68}=1.87, \mathrm{p}<0.05\right)$, although the significance level was relatively low. He showed a significant difference in the performance at different depths $\left(\mathrm{F}_{4,66}=6.33, \mathrm{p}<0.001\right)$.

Although both monkeys showed various patterns of movements at the beginning of the training, their movement pattern became rather uniform through practice. When they took food from the shallow well, they pinched the food between the thumb and digit finger. On the other hand, for deeper wells, they inserted 3 fingers (index, middle and ring) into the well at the same time to scrape the food away. They used only digit joints and rarely moved the wrist or elbow joints. After they successfully took out food, they grasped it tightly and withdrew their hands in the cage to eat it.

\section{Mapping \& cortical infarct}

As the stimulation was applied medially to laterally in the hand areas of the right M1, the muscle contraction was observed in the order of left shoulder, left hand, and digits. The distal forelimb representation (digit, wrist/forearm) was bounded laterally. Movements of proximal areas (elbow and shoulder) are represented medially. Wrist movements such as pronation and supination of the left hand were elicited. From medial to lateral hand areas, flexion and extension of little, ring, middle and index fingers were observed as shown in Fig. 3. The stimulation evoked digit movements that combined several muscles, consistent with previous reports ${ }^{14,15)}$. Experimental ischemic lesions were made in the shoulder area for Monkey A and the digit area for Monkey B as shown in Fig. 3.

\section{Hand performance after experimental infarct}

After surgery, the monkeys tended to use their unaffected right hands to retrieve food from the wells for a few days after the experimental ischemic lesions. On the 2 nd day after surgery, the monkeys were hardly able to retrieve food from the wells as before.

Monkey A showed impairments in all the joints of the contralateral upper limbs including the shoulder. From the third day after the lesion, he started to use the affected left hand. However, he was so clumsy in holding food with his left hand that he could not successfully take it to his mouth. He sometimes showed flexion of the elbow joint and even lateral flexion of the trunk to compensate for the clumsy movements of his forelimbs. Monkey A could retrieve food with his left hand on 


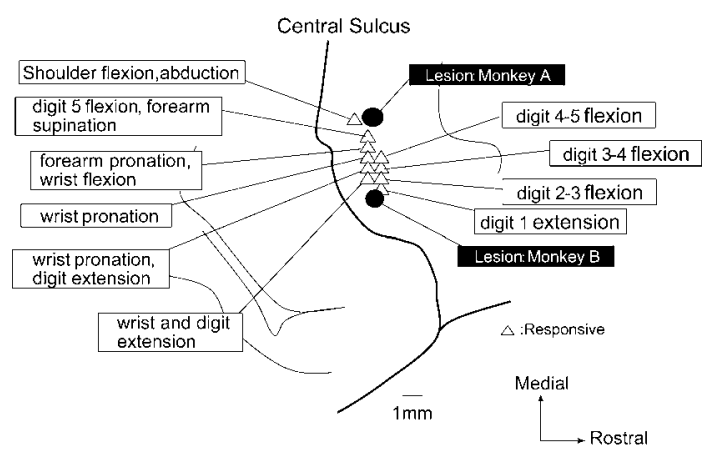

Fig. 3. The cortical map illustrating the sites of electrode penetration in the right M1 in the 2 monkeys. After stimulation, lesions were made in the 2 monkeys.

the 7th day after the lesion, but a longer time was needed as shown in Fig. $4 \mathrm{~A}\left(\mathrm{~F}_{2,253}=32.2, \mathrm{p}<0.001\right)$. There were also significant differences among the depth of the wells $\left(\mathrm{F}_{4,251}=28.5, \mathrm{p}<0.001\right)$. However, in the test sessions from the 2nd to the 6th week, he took food only with his intact right hand and did not try to use his impaired left hand. So, we were unable to obtain data during this period. Finally, on the 42nd day after the lesion, he could take food as quickly as he had done before surgery, there being no significant difference between the results of pre-surgery and those of the 6th week $(p=0.63)$. Supination or pronation of the forearm was not observed in any of the trials.

Monkey B did not show impairment in the elbow or shoulder joints after surgery, but he did show it in the wrist and digit joints. The time required for Monkey B to retrieve food was significantly increased after the lesion $\left(\mathrm{F}_{4,437}=15.0, \mathrm{p}<0.001\right)$, but decreased as the training was repeated. In the 2nd week, there was no significant difference in his results compared to that before surgery, as shown in Fig. 4B $(p=0.25)$. He showed a significant difference for the depth of the wells $\left(F_{4,437}=10.7\right.$, $\mathrm{p}<0.001$ ).

Monkey B also frequently failed to hold a piece of food in the test sessions. The percentage of failure in which Monkey B could not retrieve food at his first attempt was $50.5 \%$. On failing to hold the food, he picked it up again. The reason for the failure was lack of complete flexion and extension of the digit joints. On the 3rd day after the lesion, Monkey B moved the elbow and shoulder joints more often than before. He used flexion and extension of the elbow and abduction of the shoulder joints and even lateral flexion of the trunk during the 1st week. However, supination or pronation of the forearm was not observed in any of the trials. Monkey B showed many failures in the first and second weeks, but from the 3rd week, he succeeded in all of the trials.

Monkey B needed 14 days to reach the same level of competence as before lesion. Because Monkey A was not using his impaired hand from the 2 nd to the 6th week, we were unable to determine the accurate date of his recovery. However, he showed no significant impairment in the test sessions of the 6th week.

\section{Movement pattern analysis}

Figure 5 shows how many times each joint flexed or extended per retrieval trial in Monkey B. There were significant differences among prelesion, the 1 st week and the 1 st month $\left(\mathrm{F}_{2,324}=38.9, \mathrm{p}<0.001\right)$. However, the results at the $1 \mathrm{st}$ month did not significantly differ from those before the surgery $(p=0.69)$. This indicates the number of the joint movements per retrieval increased after the lesion, but decreased as the training progressed and reached the pre-lesion level by the 1 st month. Depth also showed significant differences in the results $\left(\mathrm{F}_{4,322}=12.0, \mathrm{p}<0.001\right)$, but the results of the $50-\mathrm{mm}$ well did not improve significantly $(\mathrm{p}=0.28)$. There was a significant difference for joints $\left(\mathrm{F}_{2,324}=26.3\right.$, $\mathrm{p}<0.001)$. However, the wrist joint did not show a significant recovery $(\mathrm{p}=0.73)$.

\section{DISCUSSION}

Although the number of monkeys was small, the present study not only confirmed that monkeys recovered with therapeutic exercises after the experimental stroke, but also showed the usefulness of the Modified Hand Performance Test with modified Klüver Board, because the compensatory movements were controlled.

\section{The usefulness of the modified Klüver board in the present study}

The test with the modified Klüver board showed less variability among the performances with the wells, compared to the results reported in previous studies $^{6,7)}$. The conventional Klüver board was used in the previous reports $6,7,13)$, and allowed the animals to use pronation and supination of the 
A. Monkey A

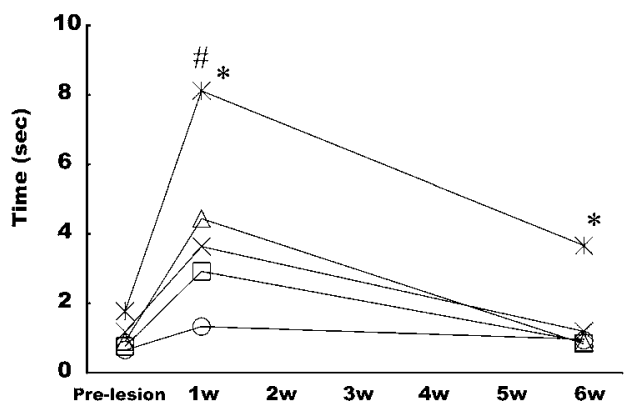

B. Monkey B

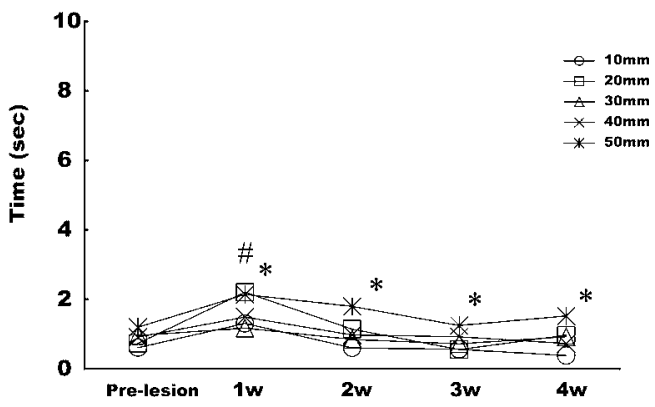

Fig. 4. The time required for taking food with the dominant hand in the Hand Performance Test for the post-lesion monkeys. A. Monkey A showed a significant difference for both days (\#) and depths $(*)$. During the period from the 1st to the 6th week, there was no data, because he did not use his impaired hand. On the 6 th week, he took food with his impaired dominant hand and the results were as fast as those of pre-lesion. The results in the wells with $50 \mathrm{~mm}$ depth showed a significant difference compared to the others (*). B. Monkey B showed significant differences for both days (\#) and depths $(*)$. When he succeeded in retrieving the food, the retrieval time was prolonged after the lesion but it recovered in the 2 nd week. The results in the well with $50 \mathrm{~mm}$ depth showed a significant difference compared to the others $(*)$.

A. Shoulder and Elbow

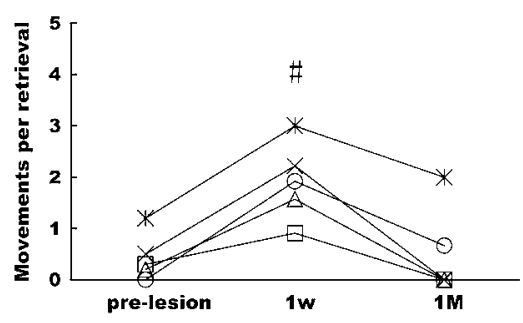

B. Wrist

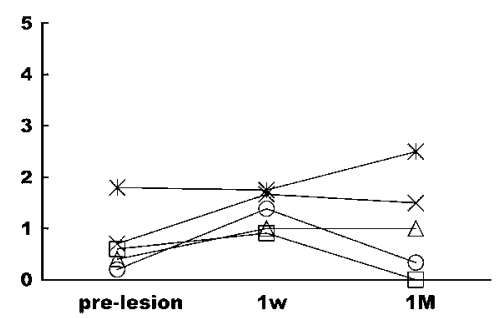

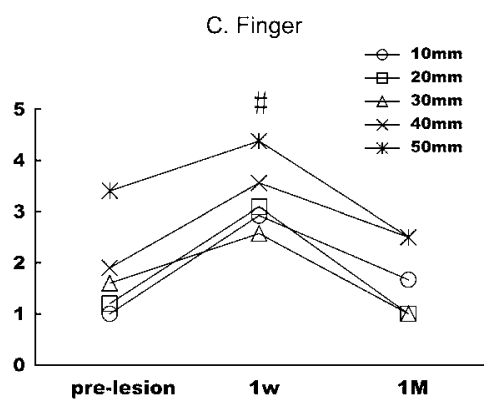

Fig. 5. The number of joint movements to retrieve food per trial with his dominant hand for Monkey B. There was a significant difference for days, depth and joints. The movement of shoulder and elbow significantly increased after surgery for each well but recovered after 1 month (\#). No significant difference was observed in wrist movements. The number of finger movements increased for each well after operation but recovered at 1 month.

forearm movements, because the sizes of the wells were $9.5,13.5,19.5$ and $25 \mathrm{~mm}$ which are relatively large for squirrel monkeys. Thus, complex movements of flexion and extension including supination and pronation of the forearm may have taken place. On the contrary, in the present study, the two monkeys showed consistent patterns of successful retrieval. This enabled us to evaluate the recovery by means of observing the numbers of flexion and extension of the joints, suggesting that the Modified Hand Performance Test of this study may be more useful for examining the hand movements involved in taking something out of the wells than the conventional one ${ }^{6,7,13)}$. It is possible that the monkeys might have wanted to take food with these compensatory movements, but the small size of wells with respect to the Monkeys' hands might inhibited them to do that. We cannot exclude the possibility that the motor commands for the compensatory movements were sent to their 
extremities but the size of the wells were too small to allow them.

One limitation of this study is that we did not perform three-dimensional recordings. Crista and Levin reported the compensatory trunk movements of stroke patients with 3-D recording ${ }^{16)}$. We cannot exclude the possibility that the monkeys showed subtle compensatory movements. However, as far as we observed with VTR, there was no supination or pronation. It is also possible that the repeated training of food retrieval controlled their performances.

In the present study, the time required for the monkeys to take food from the deepest well was significantly longer than that of the other wells. It is well known that monkeys are not good at pinching things with thumb and index fingers, because of their poor abilities to oppose the thumb ${ }^{17)}$. This is the reason why they scraped the food out with their index, middle and ring fingers from the deep wells.

The results of the present study also showed improvements of performances before the lesion in both monkeys A and B (Fig. 2B). Similar results were reported in the previous study ${ }^{18)}$. This may be due to learning effects, suggesting that cortical reorganization may occur even in intact animals.

Recovery of retrieving movements after cortical infarcts and rehabilitation training

Although the lesion in Monkey A was located in the shoulder area, the impairment extended over the left arm and digits. A lesion made by a coagulator may cause the occlusion of the blood stream of the areas of the whole arm. Both monkeys A and B showed paresis of the contralateral forelimbs, although the severity was different. Monkey A may have had severer impairments, because of his extended lesions. He needed a longer time to show recovery after the lesion (Fig. 4 A, B). However, the reason for the difference in the recovery processes in the two monkeys may not be only the severity of the impairment. The performance of Monkey A differed from that of Monkey B even before the lesion (Fig. 2), although both monkeys showed learning effects, compared to the results at the beginning (Fig. 2 A, B). This suggests the possibility that Monkey A's dominant hand may not have been so dexterous as Monkey B's from the beginning. Therefore, many factors may be involved in the differences in the results of the two monkeys.
It has been reported that therapeutic exercise facilitated the recovery of motor performances after experimental lesions in squirrel monkeys ${ }^{6,7,13)}$ and rats $^{18,19)}$. Kleim and $\mathrm{Nudo}^{19)}$ reported the changes of somatotopy in the primary motor cortex of the intact animals after they had received training of the finger movements. These reports indicate that the reorganization of the cerebral cortex corresponded to the recovery. They also suggest the possibility that the adjacent areas may be involved in recovery of the lost function. Isa et al. reported that monkeys with cervical lesions in the corticospinal tract showed high activation in the ventral premotor area in a Positron Emission Tomography (PET) study, and that the function did not change by chemical inactivation of the primary motor cortex $(\mathrm{M} 1)^{20)}$. This suggests that the ventral premotor area (area 6) may compensate for the impairment of the function of the corticospinal tract. In the present study, it is possible that the surrounding areas near the M1 such as the area 6 took over from the role of the impaired areas.

The animal model and the mechanisms of recovery

In recent years, understanding of motor learning, neuroplasticity and functional recovery after brain lesion has been growing. New findings in basic neuroscience have provided stimulus for research in motor rehabilitation. Above all, repetitive motor exercises have been reported as favorable for motor recovery in stroke patients ${ }^{21)}$. Basic neurophysiological research suggests that repetitive motor activity may form the basis of motor learning and recovery ${ }^{22)}$. Transcranial magnetic stimulation (TMS) in humans has revealed that even short-term repetition of execution in simple thumb movements induced changes in the cortical map ${ }^{23)}$.

The test in the present study made it possible to focus on a specific movement, and we confirmed the functional recovery after experimental stroke induction. Furthermore, the present study enabled us to evaluate the movement patterns of the forelimbs in monkeys, quantitatively.

Models in non-human primates have many advantages compared to the rodent models ${ }^{18)}$, because of their resemblance to humans in brain structure and function. One might doubt whether we could successfully argue the usefulness of the present study based on two monkeys' data. However, we think it possible, even with the small sample, because of less individual difference in the 
primary motor cortex in monkeys than in humans. Moreover, the lesions in the two monkeys were clear enough to evaluate, quite different from the clinical studies with patients. This animal model as those previously reported ${ }^{6,7)}$ has lesions in the cerebral cortex, while clinical strokes occur more frequently in the subcortical structure, such as the internal capsules, in humans. Despite the difference of the lesions, this animal model may be useful for future studies, which will explore how the recovery process and reorganization in the brain takes place in this model.

\section{ACKNOWLEDGEMENT}

The authors thank Dr. Kikuro Fukushima for valuable comments on this experiment. This study was supported by a Grant-in-Aid for Scientific Research from the Ministry of Education, Culture, Sport, Science and Technology of Japan (12832004).

\section{REFERENCE}

1) Macko RF, DeSouza CA, Tretter LD, et al.: Treadmill aerobic exercise training reduces the energy expenditure and cardiovascular demands of hemiparetic gait in chronic stroke patients, a preliminary report. Stroke, 1997, 28: 326-330.

2) Liepert J, Bauder H, Wolfgang HR, et al.: Treatmentinduced cortical reorganization after stroke in humans. Stroke, 2000, 31: 1210-1216.

3) Lum PS, Burgar CG, Peggy C, et al.: Robot-assisted movement training compared with conventional therapy techniques for the rehabilitation of upper-limb motor function after stroke. Arch Phys Med Rehabil, 2002, 83: 952-959.

4) Miyai I, Yagura H, Oda I, et al.: Premotor cortex is involved in restoration of gait in stroke. Ann Neurol, 2002, 52: 188-194.

5) Small SL, Hlustik P, Noll DC, et al.: Cerebellar hemispheric activation ipsilateral to the paretic hand correlates with functional recovery after stroke. Brain, 2002, 125: 1544-1557.

6) Nudo RJ, Jenkins WM, Merzenich MM, et al.: Neurophysiological correlates of hand preference in primary motor cortex of adult squirrel monkeys. J Neurosci, 1992, 12: 2918-2947.

7) Nudo RJ, Milliken GW: Reorganization of movement representations in primary motor cortex following focal ischemic infracts in adult squirrel monkeys. J
Neurophysiol, 1996, 75: 2144-2149.

8) Xerri C, Merzenich MM, Peterson BE, et al.: Plasticity of primary somatosensory cortex paralleling sensorimotor skill recovery from stroke in adult monkeys. J Neurophysiol, 1998, 79: 2119-2148.

9) Barbay S, Plautz EJ, Friel KM, et al.: Behavioral and neurophysiological effects of delayed training following a small ischemic infarct in primary motor cortex of squirrel monkeys. Exp Brain Res, 2006, 169: 106-116.

10) Brunnstrom $S$ : Motor testing procedures in hemiplegia. J Am Phys Ther Assoc, 1966, 46: 357-375.

11) Friel KM, Nudo RJ: Recovery of motor function after focal cortical injury in primates: compensatory movement patterns used during rehabilitative training. Somatosensory \& Motor Research, 1998, 15: 173-189.

12) Bobath B: Principles of treatment. In: Adult Hemiplegia: Evaluation and Treatment. London: William Heinemann Medical Books, 1978, p 59.

13) Lawrence DG, Kuyper HGJM: The functional organization of the motor system in the monkey. The effects of bilateral pyramidal lesions. Brain, 1968, 91: $1-33$.

14) Sato KC, Tanji J: Digit-muscle response evoked from multiple intracortical foci in monkey precentral motor cortex. J Neurophysiol, 1989, 62: 959-969.

15) Penfield W, Rasmussen $T$ : The Cerebral Cortex of Man. A Clinical Study of Localization of Function. New York: Macmillan, 1950.

16) Cristea MC, Levin MF: Compensatory strategies for reaching in stroke. Brain, 2000, 123: 940-953.

17) Howell $A B$, Straus $W$ : The brachial flexior muscles in primates. PNAS, 1931, 80: 1-31.

18) Kleim JA, Barbay S, Nudo RJ: Functional reorganization of the rat motor cortex following motor skill learning. J Neurophysiol, 1998, 80: 3321-3325.

19) Bury SD, Jones TA: Unilateral sensorimotor cortex lesions in adult rats facilitate motor skill learning with the "unaffected" forelimb and training-induced dendritic structural plasticity in the motor cortex. J Neurosci, 2002, 22: 8597-8606.

20) Isa $T$ : What can we learn from the functional recovery after brain injury? In: Proc. the 29th Annual meeting of Japan Neuroscience Society. 2006, TS2-1-1.

21) Woldag $\mathrm{H}$, Hummelsheim $\mathrm{H}$ : Evidence-based physioltherapeutic concepts for improving arm and hand function in stroke patients. J Neurol, 2002, 249: 518-528.

22) Asanuma H, Pavlides $\mathrm{C}$ : Neurobiological basis of motor learning in mammals. review. Neuroreport, 1997, 8: i-vi.

23) Classen J, Liepert J, Wise SP, et al.: Rapid plasticity of human cortical movement representation induced by practice. J Neurophysiol, 1998, 72: 1117-1123. 